\title{
Biochemical characterization of ParI, an orphan C5-DNA methyltransferase from Psychrobacter arcticus 273-4
}

\author{
Miriam Grgic ${ }^{\mathrm{a}}$, Adele Williamson ${ }^{\mathrm{a}}$, Gro Elin Kjæreng Bjerga ${ }^{\mathrm{b}}$, Bjørn Altermark ${ }^{\mathrm{a}}$, Ingar Leiros ${ }^{\mathrm{a}, *}$ \\ a The Norwegian Structural Biology Centre (NorStruct), Department of Chemistry, Faculty of Science and Technology, UiT the Arctic University of Norway, N-9037, Troms $\phi$, Norway \\ ${ }^{\mathrm{b}}$ Uni Research, Center for Applied Biotechnology, Thormohlens Gate 55, N-5006, Bergen, Norway
}

\section{A R T I C L E I N F O}

Keywords:

DNA methylation

DNA methyltransferase

ParI

Psychrobacter arcticus 273-4

Phage origin

\begin{abstract}
A B S T R A C T
Cytosine-specific DNA methyltransferases are important enzymes in most living organisms. In prokaryotes, most DNA methyltransferases are members of the type II restriction-modification system where they methylate host DNA, thereby protecting it from digestion by the accompanying restriction endonucleases. DNA methyltransferases can also act as solitary enzymes having important roles in controlling gene expression, DNA replication, cell cycle and DNA post-replicative mismatch repair. They have potential applications in biotechnology, such as in labeling of biopolymers, DNA mapping or epigenetic analysis, as well as for general DNA-protein interaction studies.

The parI gene from the psychrophilic bacterium Psychrobacter arcticus 273-4 encodes a cytosine-specific DNA methyltransferase. In this work, recombinant ParI was expressed and purified in fusion to either an N-terminal hexahistidine affinity tag, or a maltose binding protein following the hexahistidine affinity tag, for solubility improvement. After removal of the fusion partners, recombinant ParI was found to be monomeric by size exclusion chromatography, with its molecular mass estimated to be $54 \mathrm{kDa}$. The apparent melting temperature of the protein was $53^{\circ} \mathrm{C}$ with no detectable secondary structures above $65^{\circ} \mathrm{C}$. Both recombinant and native ParI showed methyltransferase activity in vivo. In addition, MBP- and His-tagged ParI also demonstrated in vitro activity. Although the overall structure of ParI exhibits high thermal stability, the loss of in vitro activity upon removal of solubility tags or purification from the cellular milieu indicates that the catalytically active form is more labile. Horizontal gene transfer may explain the acquisition of a protein-encoding gene that does not display common cold-adapted features.
\end{abstract}

\section{Introduction}

The methylated DNA bases N4-methylcytosine, N6-methyladenine and C5-methylcytosine are considered additional bases of the genetic code that carry epigenetic information not encoded in the DNA sequence itself (reviewed in Refs. [1] and [2]). Generally, DNA methylation has an important role in protein-DNA interaction by either enhancing or disrupting binding of proteins to DNA. The enzymes responsible for these modifications are DNA methyltransferases (DNA-MTases), which catalyze the transfer of a methyl group from S-adenosyl-L-methionine (SAM) to the three above-mentioned positions in DNA [3]. In contrast to the role of eukaryotic DNA-MTases, most prokaryotic DNA-
MTases are members of a host protection system, the restriction-modification (RM) system [4,5]. The main function of DNA-MTases in the RM system is methylation of host DNA which confers protection from digestion by restriction endonucleases (REases) that recognize the same specific DNA sequence [6]. Some DNA-MTases are not accompanied by an REase and are so-called orphan MTases [7]. The roles of orphan MTases have been proposed to be within gene regulation, DNA replication, cell cycle and directing post-replicative mismatch repair on newly synthesized DNA strands by de novo methylation [2,8-12].

In contrast to eukaryotic DNA 5-cytosine methyltransferases (C5-DNA-MTases), being multi-domain proteins and complexes, the prokaryotic enzymes are single-domain proteins [13]. When three-dimensional structures of C5-DNA-MTases are compared, both prokary-

Abbreviations: TEV, tobacco etch virus; DSC, differential scanning calorimetry; CD, circular dichroism; DNA-MTase, DNA methyltransferase; C5-DNA-MTase, DNA 5-cytosine methyltransferase; MBP, maltose binding protein.

* Corresponding author.

Email addresses: miriam.grgic@uit.no (M. Grgic); adele.k.williamson@uit.no (A. Williamson); Gro.Bjerga@uni.no (G.E. Kjæreng Bjerga); bjorn.altermark@uit.no (B. Altermark); ingar. leiros@uit.no (I. Leiros) 
otic and eukaryotic catalytic DNA-MTase domains are similar in structural organization. The catalytic domains are organized into a large and a small sub-domain separated by a marked cleft [14]. Despite their structural homology, the sequence similarity among C5-DNA-MTases is low with the exception of ten conserved motifs, named with roman numerals I-X [3]. In addition to these motifs, all C5-DNA-MTases possess a variable region; the Target Recognition Domain (TRD), which is involved in sequence recognition [3,9]. Most of the conserved motifs are located in the large sub-domain, while the TRD comprises most of the small sub-domain. The best conserved motifs (I, IV, VI, VIII, IX and X) are either structural (motif IX), or involved in SAM binding (motifs I and X), DNA binding (motifs VI, VIII and TRD) or catalysis (motif IV) [3,14-16].

Psychrobacter arcticus 273-4 is a gram-negative bacterium discovered in 20-30 thousand year old permafrost soil in Kolyma, Siberia [17]. $P$. arcticus 273-4 has been genome sequenced [18], grows at temperatures from -10 to $28^{\circ} \mathrm{C}$ and has generation time of 3.5 days at temperatures below zero [17-20]. At the protein level, P. arcticus 273-4 possesses many common features for psychrophilic bacteria, such as reduced use of proline, arginine and acidic amino acids, an increased lysine content, as well as encoding several cold shock proteins [18-21].

In the present study, a C5-DNA-MTase from P. arcticus 273-4, ParI, was characterized on the basis of its potential to possess features relevant for biotechnological applications, such as labeling of biopolymers, DNA mapping or epigenetic analysis [22-24].

\section{Materials and methods}

\subsection{Bioinformatic analyses}

A multiple protein sequence alignment where ParI was compared to bacterial C5-DNA-MTase homologs [HhaI from Haemophilus parahaemolyticus (GI: 127455); M. SssI from Spiroplasma sp. (GI: 417325); AbaI from Acinetobacter baumannii UH5107 (GI: 446969424) ] and the catalytic domain of human DNMT1 (GI: 12231019) was made using the T-Coffee web server [25]. The sequence alignment was rendered with the ESPript server [26]. The sequence alignment was annotated with secondary structure of HhaI (PDB ID: 1MHT) and ParI secondary structure predictions from the PsiPred server [27]. The genomic context of ParI in P. arcticus 273-4 was analysed by the PHAST server [28]. Promoter prediction was performed with BPROM [29], while the Rho-independent terminators were predicted by ARNold [30,31].

\subsection{Cloning, expression and purification}

The gene encoding ParI, parI [GenBank ID: 71038525] was amplified from $P$. arcticus 273-4 (DSMZ) with primers synthesized by Sigma-Aldrich and Phusion polymerase (NEB) in a PCR reaction following the protocol for Phusion polymerase. The amplified gene, including a primer-encoded TEV-cleavage site, was cloned into either the pDest17 vector which encodes a His tag (resulting in pHis-ParI), or the pHMGWA vector encoding an N-terminal hexahistidine (His) tag and maltose binding protein (MBP). Both constructs were cloned using the Gateway technology (Thermo Fisher Scientific), according to the protocol provided by the manufacturer [32]. All primers used are listed in Tables S-2. Newly cloned constructs were transformed by the heat-shock method into the McrBC-negative E. coli T7 Express strain (NEB) for recombinant expression and purification (genotype fhuA2 lacZ::T7 gene1 [lon] ompT gal sulA11 R(mcr-73::miniTn10--TetS)2 [dcm] $R$ (zgb-210::Tn10--TetS) endA1 $\Delta$ (mcrC- mrr)114::IS10). The protein was expressed in $1 \mathrm{~L}$ cultures of Lysogeny broth (LB) media and induced with isopropyl- $\beta$-D-1-thiogalactopyranoside (IPTG, VWR) at a final concentration of $0.5 \mathrm{mM}$. Cells for both His-ParI and His-MBP-tagged ParI were harvested after $7 \mathrm{~h}$ expression at $20^{\circ} \mathrm{C}$, by centrifugation at $7500 \times g$ for 30 minat room temperature and resuspended in lysis buffer ( $50 \mathrm{mM}$ Tris $\mathrm{pH} 8.0,750 \mathrm{mM} \mathrm{NaCl}, 5 \%$ (v/v) glycerol, $10 \mathrm{mM} \mathrm{MgCl}_{2}$ ) supplemented with $1 \mathrm{x}$ Complete protease inhibitor cocktail (Roche). The cells were disrupted by a cell disruptor (Constant Systems, Ltd.) using a pressure of $1.35 \mathrm{kbar}$ in four cycles. The lysate was cleared by centrifugation at $20000 \times g$ for 30 minat $4^{\circ} \mathrm{C}$ after which it was incubated with HL/SAN DNase (ArticZymes) for $1 \mathrm{~h}$ at $4^{\circ} \mathrm{C}$. Affinity purification of recombinant His-ParI or His-MBP-ParI was carried out on a $5 \mathrm{ml}$ HisTrap HP column (GE Healthcare) equilibrated with buffer A $(50 \mathrm{mM}$ Tris $\mathrm{pH}$ $8.0,750 \mathrm{mM} \mathrm{NaCl}, 5 \%(\mathrm{v} / \mathrm{v})$ glycerol and $10 \mathrm{mM}$ imidazole) using an ÄKTA purifier (GE Healthcare). The bound protein was eluted across a gradient of $0-100 \%$ buffer B (50 mM Tris $\mathrm{pH} 8.0,750 \mathrm{mM} \mathrm{NaCl}, 5 \%$ (v/ v) glycerol and $500 \mathrm{mM}$ imidazole). The purity of the protein was evaluated by SDS-PAGE. In the case of His-MBP-tagged ParI, the removal of the fusion partner was carried out in buffer C ( $50 \mathrm{mM}$ Tris $\mathrm{pH} 8.0$, $200 \mathrm{mM} \mathrm{NaCl}, 5 \%$ (v/v) Glycerol, $1 \mathrm{mM}$ DTT and $0.5 \mathrm{mM}$ EDTA) supplemented with TEV protease in a $1: 10 \mathrm{mg} / \mathrm{mg}$ ratio to ParI and incubated overnight at $4{ }^{\circ} \mathrm{C}$. Buffer exchange was done using a HiPrep 26/60 desalting column (GE Healthcare). The recombinant ParI protein was recovered from the flow-through after a second step of HisTrap affinity purification, while the His-MBP portion remained bound to the column. To determine the oligomeric state of the protein, untagged ParI was separated on a HiLoad 16/60 Superdex 200 prep grade gel filtration chromatography column (GE Healthcare) in buffer C. The protein concentration was determined by measuring the absorbance at $280 \mathrm{~nm}$ using a Nanodrop spectrophotometer (NanoDrop Technologies). The theoretical extinction coefficient for the protein is $63995 \mathrm{M}^{-1} \mathrm{~cm}^{-1}$ as calculated by the ProtParam tool. ${ }^{1}$ Pure protein was concentrated by centrifugation through $10 \mathrm{kDa}$ MWCO Amicon Ultra Centrifugal filters (Merck Millipore). The identity of recombinant protein was confirmed by MS/MS by the Proteomics facility (UiT, The Arctic University of Norway).

\subsection{Thermofluor assay}

To assess the stability of ParI, a fluorescence-based thermal shift (Thermofluor) assay was used [33]. A buffer screen consisting of 24 different buffers at various pHs was performed. (Bicine, $\mathrm{pH}$ 8; Bicine, $\mathrm{pH}$ 9; Hepes, pH 7; Hepes, pH 7.5; Hepes, $\mathrm{pH}$ 8; Imidazole, $\mathrm{pH} 8$; MES, $\mathrm{pH}$ 6; MES, $\mathrm{pH}$ 6.2; MES, $\mathrm{pH}$ 6.5; Potassium phosphate, $\mathrm{pH}$ 5; Potassium phosphate, $\mathrm{pH}$ 6; Potassium phosphate, $\mathrm{pH} 7$; Sodium acetate, $\mathrm{pH}$ 4.5; Sodium acetate, $\mathrm{pH}$ 5; Sodium cacodylate, $\mathrm{pH}$ 6.5; Sodium citrate, $\mathrm{pH}$ 4.7; Sodium citrate, $\mathrm{pH}$ 5.5; Sodium phosphate, $\mathrm{pH}$ 5.5; Sodium phosphate, $\mathrm{pH}$ 6.5; Sodium phosphate, $\mathrm{pH}$ 7.5; Tris, $\mathrm{pH}$ 7.5; Tris, $\mathrm{pH}$ 8; Tris, $\mathrm{pH}$ 8.5). The final concentration of protein in the reaction was $0.5 \mathrm{mg} /$ ml. SYPRO ${ }^{\circledR}$ Orange Protein Gel Stain (Sigma-Aldrich) was diluted 1:75 from the starting concentration. The assay was performed in a volume of $25 \mu \mathrm{l}$ and was run in a MiniOpticon real-time PCR system (BioRad) in a temperature range from $5^{\circ} \mathrm{C}$ to $95^{\circ} \mathrm{C}$ with $1{ }^{\circ} \mathrm{C}$ increment every $3 \mathrm{~s}$.

\subsection{Differential scanning calorimetry}

Thermal denaturation curves were recorded at temperatures between $5^{\circ} \mathrm{C}$ and $95^{\circ} \mathrm{C}$ using a scan rate of $1^{\circ} \mathrm{C} / \mathrm{min}$ in an $\mathrm{N}$-DSC III calorimeter (Calorimetry sciences corp.). ParI was dialyzed against DSC-buffer (50 mM HEPES pH 8.0, $200 \mathrm{mM} \mathrm{NaCl)}$ and used at a final

\footnotetext{
1 Expasy server: http://web.expasy.org/protparam/.
} 
concentration of $1.4 \mathrm{mg} / \mathrm{ml}$. The DSC-buffer from dialysis was used as a reference.

\subsection{Circular dichroism}

ParI was dialyzed overnight at $4{ }^{\circ} \mathrm{C}$ against CD-buffer $(10 \mathrm{mM}$ Tris $\mathrm{pH} 8.0$ and $100 \mathrm{mM} \mathrm{NaF}$ ). The samples were filtered through a $0.45 \mu \mathrm{m}$ pore size filter (Spin X Costar) to remove precipitate and diluted to a final concentration of $0.15 \mathrm{mg} / \mathrm{ml}$. SAM was added at a final concentration of $200 \mu \mathrm{M}$ to either protein or buffer directly before measurement. Data was collected on a J-810 CD spectrophotometer (Jasco) using a $1 \mathrm{~mm}$ path length cuvette and the following settings: sensitivity 100 mdeg, datapitch $0.5 \mathrm{~nm}$, scan speed $50 \mathrm{~nm} / \mathrm{min}$, response $2.0 \mathrm{~s}$, bandwidth $1 \mathrm{~nm}$, accumulation three scans, units CD mdeg. Three scans were recorded and averaged. Three scans of buffer were also recorded in absence or presence of SAM. The measurements were done at $15^{\circ} \mathrm{C}, 35^{\circ} \mathrm{C}$ and $65^{\circ} \mathrm{C}$. The measurement at $15^{\circ} \mathrm{C}$ was done in the presence and absence of SAM, while measurements at $35^{\circ} \mathrm{C}$ and $65^{\circ} \mathrm{C}$ were performed without SAM. In the data analysis, each set of spectra corresponding to each condition was analysed with two different programs, using the SP170 reference set [34-41]. Data analysis was conducted through the Dichroweb server [39].

\subsection{In vivo MTase genome assay}

To investigate His-ParI activity, $P$. arcticus was cultivated in LB media at $15^{\circ} \mathrm{C}$ for 3 days while the methylase-deficient control, a closely related mesophilic Psychrobacter sp. PRwf-1, which lacks a C5-DNA-MTase, was cultivated at $37^{\circ} \mathrm{C}$ overnight. Recombinant ParI activity was determined by transforming $50 \mu \mathrm{l}$ cultures of McrBC-negative E. coli T7 Express strain (NEB) with the pHis-ParI construct. Cells were grown until late log phase (OD 0.7-0.9) before induction of recombinant protein with IPTG, and further cultivation at $20^{\circ} \mathrm{C}$ for $7 \mathrm{~h}$. Untransformed T7 Express cells were treated in the same manner and served as a negative control. Genomic DNA from all bacteria was extracted using the GenElute Gel extraction Kit (Sigma Aldrich), and treated with McrBC endonuclease (NEB) in 1x NEB2 buffer (NEB) supplemented with $200 \mu \mathrm{g} / \mathrm{ml} \mathrm{BSA}$ and $1 \mathrm{mM} \mathrm{GTP}$, and incubated at $37^{\circ} \mathrm{C}$ for $1 \mathrm{~h}$. The reaction mix was separated by $1 \%$ agarose gel electrophoresis and the DNA was post-stained with RedSafe (iNtRON Biotechnology). Digestion by McrBC relative to the negative controls was taken as an indication of DNA methylation by ParI. As a positive control, pUC19 vector methylated with M. SssI methyltransferase (New England Biolabs) was used.
In a second in vivo assay, adapted from Ref. [42], the pHis-ParI construct was transformed into two different $E$. coli expression strains, BL21Star (DE3) (Life Technologies) and the McrBC-negative T7 Express (NEB). Cells were transformed as previously described and plated on LB plates containing $100 \mu \mathrm{g} / \mathrm{ml}$ ampicillin and $0.2 \mathrm{mM}$ IPTG. The plates were incubated at $37^{\circ} \mathrm{C}$ overnight.

\subsection{In vitro methyltransferase activity assay with radiolabelled SAM (3H- SAM)}

The reactions were performed with 25ng DNA (pUC19 vector), $1 \mathrm{mg} / \mathrm{ml}$ of either purified proteins (His-MBP-ParI, His-ParI and ParI) or clarified lysates (His-MBP-ParI (lysate) and His-ParI (lysate)) and $14 \mu \mathrm{M} 3 \mathrm{H}-\mathrm{SAM}$ in methyltransferase buffer (NEB2 buffer: $50 \mathrm{mM} \mathrm{NaCl}$, $10 \mathrm{mM}$ Tris- $\mathrm{HCl}, 10 \mathrm{mM} \mathrm{MgCl}_{2}, 1 \mathrm{mM}$ DTT), adjusted to a total volume of $20 \mu \mathrm{l}$ with nuclease-free $\mathrm{H}_{2} \mathrm{O}$. The samples were incubated at RT for 120 min. After incubation, reactions were spotted onto Filtermat A, 24 Well (PerkinElmer). Filters were then washed three times with 50\% TCA and once with 100\% Ethanol. The filters were dried and analysed by liquid scintillation counting (MicroBeta2, 2450 microplate counter, PerkinElmer) using scintillation liquid (Ultima Gold XR, PerkinElmer); reading $1 \mathrm{~min}$ per well. The same procedure was followed for M. SssI which was used as a positive control in the experiments, although not included in the results as its molar concentration was unknown. All reactions were blank-corrected against the same setup with no protein added. 3H-SAM was purchased from PerkinElmer. M. SssI and NEB2 buffer were purchased from New England Biolabs. The presented results are triplicates of each sample. Differences in molar protein concentration between the constructs were adjusted for.

\section{Results}

\subsection{Bioinformatic analyses of the parI gene}

To investigate the genetic context of ParI, analysis of the RM complement of the $P$. arcticus 273-4 genome and prediction of prophage-containing regions were performed. Through queries in the REBASE database of restriction enzymes [5], several putative RM systems in the $P$. arcticus 273-4 genome were identified, including one complete, but uncharacterized type I RM system, as well as two solitary RM enzymes homologous to type II DpnI-like REases and C5-DNA-MTases (ParI), respectively (Table 1). However, no interaction partners that would constitute functional type II RM systems were identified for either of these two solitary enzymes.

Table 1

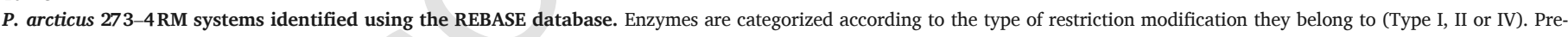

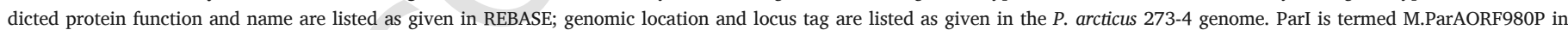
REBASE.

\begin{tabular}{|c|c|c|c|c|}
\hline Type & Function $^{\mathrm{a}}$ & Name $^{\mathrm{b}}$ & Genomic location (bp) & Locus tag \\
\hline \multirow[t]{8}{*}{ I } & M & M.ParAORF415AP & 517547-517894 & Psyc_0413 \\
\hline & M & M.ParAORF415BP & $518942-520957$ & Psyc_0415 \\
\hline & S & S.ParAORF415P & $520957-522330$ & Psyc_0416 \\
\hline & $\mathrm{R}$ & ParAORF415P & $528255-531563$ & Psyc_0424 \\
\hline & $\mathrm{R}$ & ParAORF723P & $862503-863966$ & Psyc_0724 \\
\hline & M & M.ParAORF877P & 1044227-1045816 & Psyc_0877 \\
\hline & $S$ & S.ParAORF877P & $1045806-1047080$ & Psyc_0878 \\
\hline & $\mathrm{R}$ & ParAORF877P & $1049066-1052248$ & Psyc_0881 \\
\hline \multirow[t]{4}{*}{ II } & $\mathrm{R}$ & ParAORF142P & 169265-170029 & Psyc_0143 \\
\hline & M & M.ParAORF401P & $500671-502233$ & Psyc_0401 \\
\hline & $\mathrm{R}$ & ParAORF401P & $502506-503840$ & Psyc_0402 \\
\hline & M & M.ParAORF980P & $1175124-1176590$ & Psyc_0980 \\
\hline IV & $\mathrm{R}$ & ParAMcrBP & $266672-268168$ & Psyc_0226 \\
\hline
\end{tabular}

a Predicted protein functions are listed as Restriction (R), Modification (M) or Specificity (S).

b As given in REBASE. 
Further investigation of the $P$. arcticus 273-4 genome in REBASE indicated that parI (locus tag Psyc_0980) is part of an operon consisting of four genes from locus tag Psyc_0981 to locus tag Psyc_0978, the rest of which are designated as hypothetical proteins (Fig. 1). The assignment of these genes as an operon is further supported by the prediction of a promoter in front of the gene from locus tag Psyc_0981 and two Rho-independent terminators after the gene with locus tag Psyc_0978 (Fig. 1) which would be capable of regulating transcription of the gene cluster. The genome of $P$. arcticus contains three prophage regions, including one complete temperate phage and two regions where phage genes are clustered (Tables S-1). Although the parI gene was not found to be part of any of these prophage regions, the flanking genes were identified as being of phage origin. Previously, two almost complete phage genomes in the $P$. arcticus genome were identified [18], the second of which is located between bp 1177957 and 1223710 and is most similar to the genome of a $45.7 \mathrm{~kb}$ MU phage. This region is directly downstream of parI and encodes the genes with locus tags Psyc_0981 and Psyc_0982.

Additional BLAST searches of the individual genes from the cluster showed that the gene with locus tag Psyc_0979 has strong nucleotide sequence similarity (82\%) to a gene from a temperate bacteriophage Psymv2 isolated from Psychrobacter sp. MV2 which indicates that these genes may have been horizontally transferred and are of phage origin, although they are no longer part of an intact temperate phage [43].

\subsection{Sequence homology of ParI to other methyltransferases}

A multiple sequence alignment of the $P$. arcticus 273-4 ParI protein sequence with other C5-DNA-MTase sequences identified most of the conserved motifs specific for these enzymes (Fig. 2). In motif IV, the PCQ catalytic sequence, which is generally highly conserved among C5-DNA-MTases [14], is replaced by DCK in ParI. BLAST searches revealed that ParI shared the highest sequence similarity (64\%) to an uncharacterized C5-DNA-MTase from Acinetobacter baumannii UH5107, which also possesses the same PCQ-to-DCK substitution in motif IV. This ParI homologue in A. baumannii is located in a region that is recognized as a phage region by PHAST. Both these bacteria belong to the same family, Moraxellaceae, which may explain the high sequence similarity [44].

\subsection{Expression and purification of recombinant ParI}

The $m c r$ locus has previously been shown to reduce recovery of methylated sequences from an organism containing methylated cytosines [45]. DNA methylated by active recombinant ParI would be a substrate for digestion by the McrBC gene product. For this reason, an McrBC-negative E. coli strain was selected for Parl expression.

Recombinant N-terminally tagged ParI (His-ParI) was successfully produced and purified in small amounts (Fig. 3A), however the yield was extremely low relative to native $E$. coli proteins meaning that large culture volumes (up to $8 \mathrm{~L}$ ) were necessary to obtain mg quantities of pure protein. Furthermore, His-ParI appeared to be very unstable and was prone to aggregation during up-concentration to $>1 \mathrm{mg} / \mathrm{ml}$. Nei- ther longer cultivation times (overnight) nor use of autoinduction media improved yields, both resulting in un-detectable His-ParI expression (data not shown). To increase protein yields to quantities suitable for biophysical characterization, ParI was produced in fusion to an $\mathrm{N}$-terminal His-tag and maltose binding protein (His-MBP), the latter of which is known to promote solubility (Fig. 3B) [46,47]. The MBP fusion partner was successfully removed by treatment with tobacco etch virus (TEV) protease (Fig. 3B), although there was a substantial loss of ParI during subsequent chromatographic purification steps after MBP-tag removal. This method typically yielded $2.5 \mathrm{mg}$ of untagged ParI per L of expression culture. Size exclusion chromatography coincided with the theoretical protein molecular weight of $54 \mathrm{kDa}$ indicating the protein is a monomer in solution (Fig. 4). No DNA contamination was observed in the purified protein based on its absorption at $260 \mathrm{~nm}$.

\subsection{Thermal stability of ParI}

As initial experiments showed that there was a substantial loss of soluble protein during purification, thermal denaturation assays were conducted to assess the general stability of ParI in a variety of buffer conditions. Among the tested buffers, HEPES pH 8.0 was determined to be the most stabilizing. ParI unfolding in HEPES at $\mathrm{pH}$ 8.0, monitored using the Thermofluor assay [33], indicates a melting temperature of $53^{\circ} \mathrm{C}$. In this experimental condition the melting curve was broad, stretching over a temperature range of $45-65^{\circ} \mathrm{C}$, which is typically observed in partially unfolded proteins [33].

The thermal denaturation temperature was supported by differential scanning calorimetry (DSC) measurements of ParI, which indicated that this construct unfolds in a single transition event with a melting temperature of $54^{\circ} \mathrm{C}, \Delta \mathrm{H}$ of $82.81 \mathrm{kcal} / \mathrm{mol}$ and $\Delta S$ of $0.2411 \mathrm{kcal} /(\mathrm{mol} \mathrm{K})$ (Fig. 5A). The range of the unfolding transition measured by DSC was $49-59^{\circ} \mathrm{C}$, which is consistent with the Thermofluor results. Thermal denaturation of ParI was found to be irreversible, and after the experiment, precipitated protein was observed in the calorimetric cell.

To further investigate the integrity of ParI, we recorded circular dichroism (CD) spectra (Fig. 5B) [48]. Our analyses indicate that both at $15^{\circ} \mathrm{C}$ and $35^{\circ} \mathrm{C}$ ParI comprises $20 \% \alpha$-helix 30\% $\beta$-strand and $12 \%$ turns, while the remaining $35-40 \%$ of the structure are disordered loops and turns. This is consistent with the secondary structure content predicted by the PsiPred server (Fig. 2). The CD spectrum of ParI at $65^{\circ} \mathrm{C}$ was typical for unfolded protein; no secondary structures were observed, which correlates with the Thermofluor and DSC measurements (Fig. 5B). Furthermore, no change in secondary structure was observed in CD measurements at $15^{\circ} \mathrm{C}$ in the presence of SAM, indicating that binding of SAM does not induce major structural changes in ParI, or possibly that the recombinant protein was defective in SAM binding.

Thus, three independent biophysical methods indicate that ParI is in a fully folded state with intact secondary and tertiary structure at temperatures below $35^{\circ} \mathrm{C}$, and that it denatures at temperatures above $53^{\circ} \mathrm{C}$.

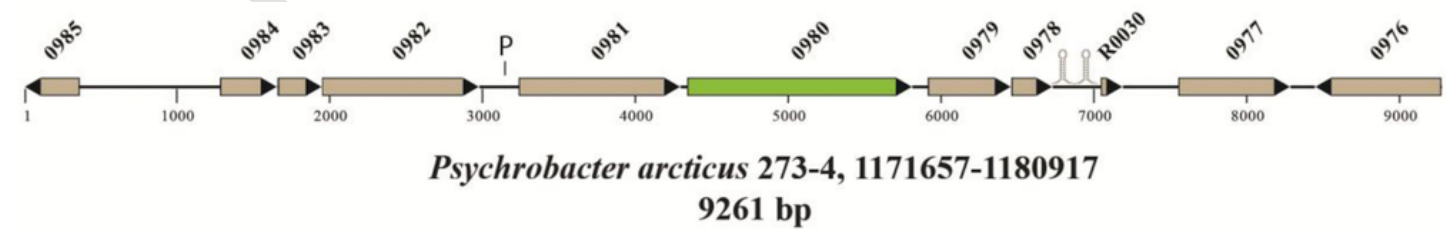

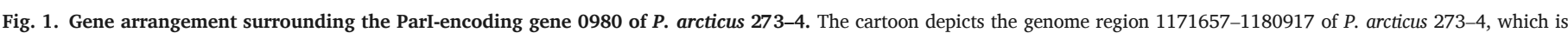

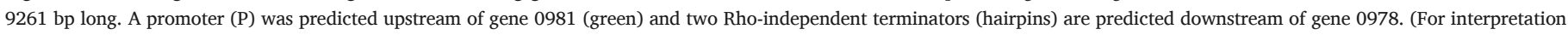
of the references to colour in this figure legend, the reader is referred to the Web version of this article.) 

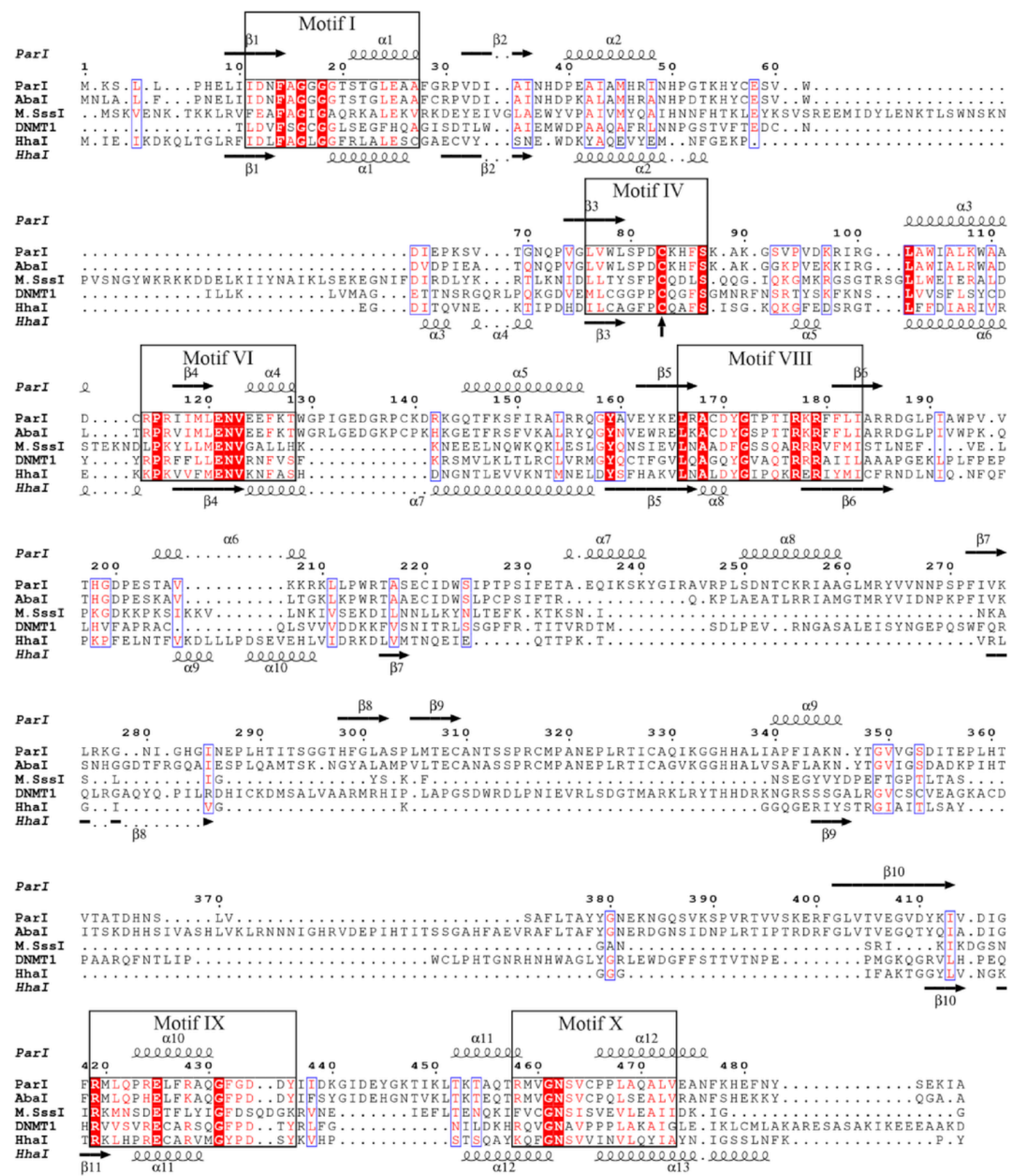

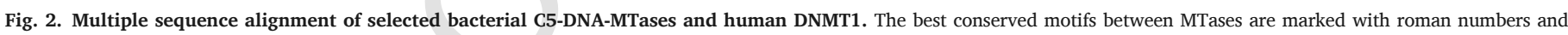

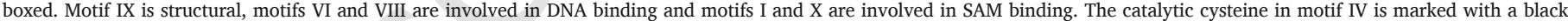

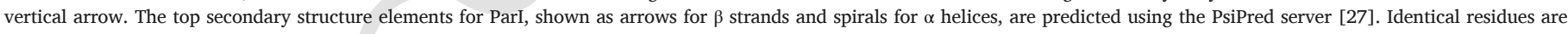

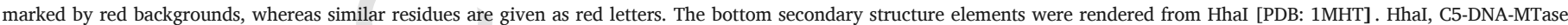

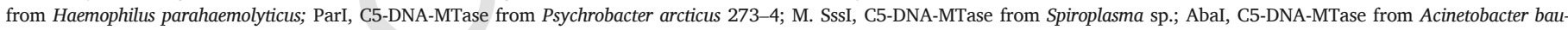

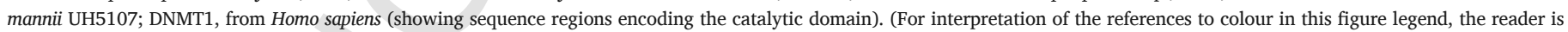
referred to the Web version of this article.)

\subsection{Both recombinant and native ParI possess methyltransferase activity in} vivo

To determine whether the recombinant His-ParI was active, we used an in vivo assay where we screened for McrBC sensitivity of genomic DNA in the presence or absence of ParI. Briefly, genomic DNA was extracted from cultures of MTase-deficient E. coli that had been transformed with a plasmid encoding the gene for His-ParI. Genomic DNA extracted from these His-ParI-expressing E. coli was digested upon treatment with McrBC, whereas genomic DNA from the ParI-deficient control cells (transformed with empty vector) remained intact (Fig. 6). As McrBC digests both hemi- and fully-methylated DNA, this result strongly suggests that recombinant His-ParI exhibits methyltransferase activity in vivo.

Transformation of the plasmid encoding His-tagged ParI into regular E. coli BL21Star (DE3) produced no colonies when grown on agar plates, supplemented with IPTG for induction; while if the plasmid was transformed to McrBC-negative E. coli, many colonies were observed (460 and 580 in two independent experiments). We suggest that the lack of growth in regular $E$. coli is caused by ParI methylation of the genomic DNA, which is subsequently digested by the McrBC system lead- 

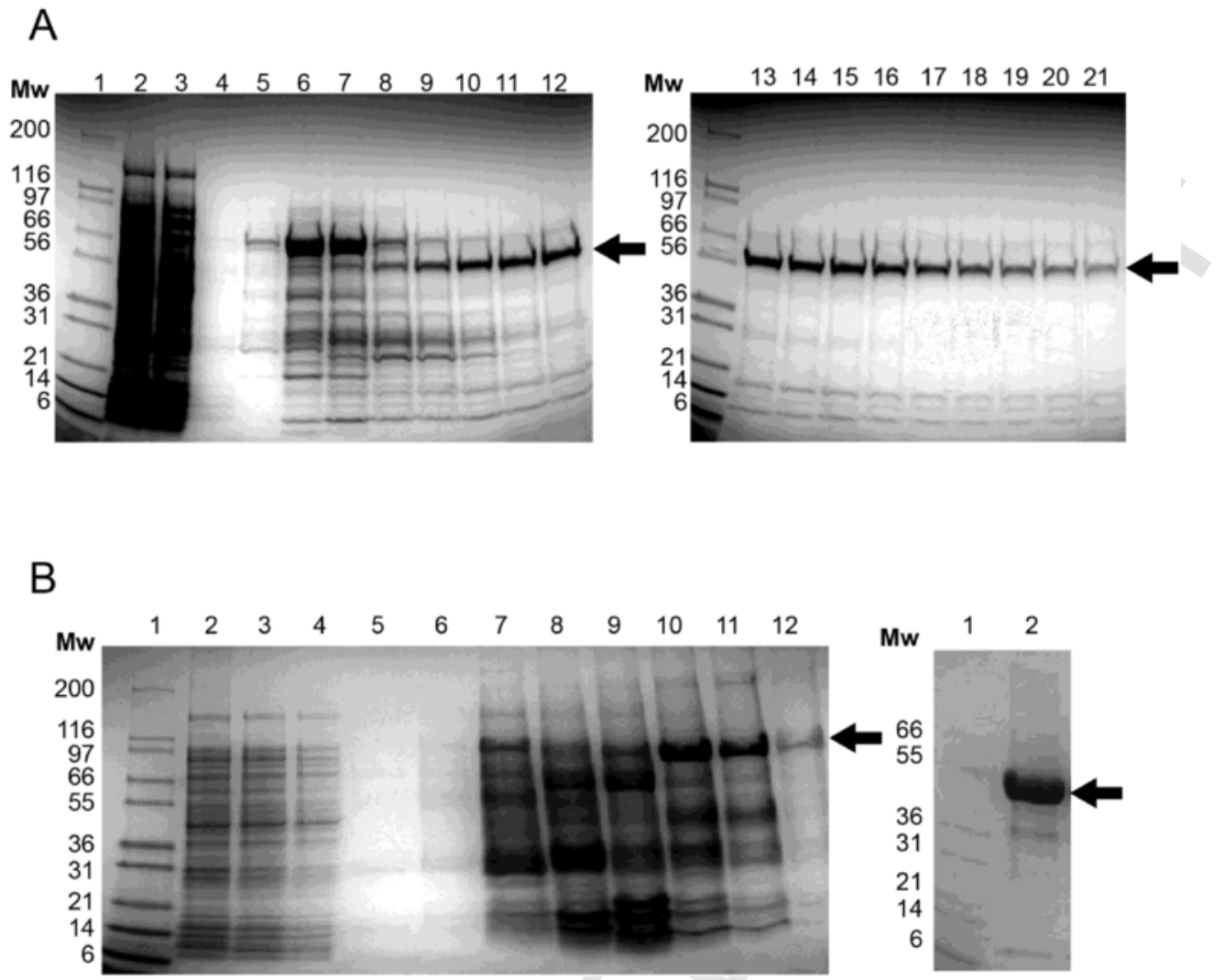

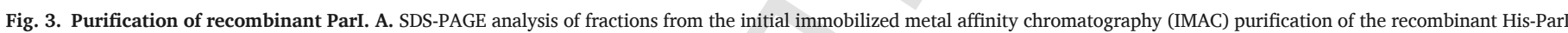

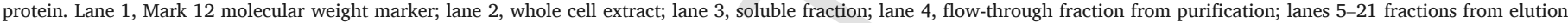

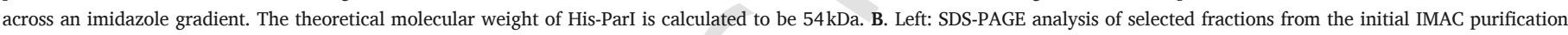

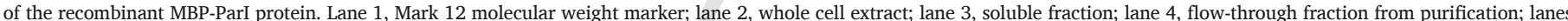

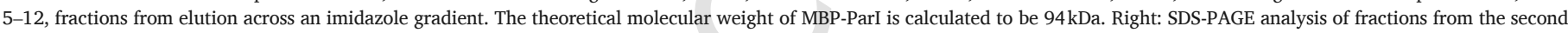

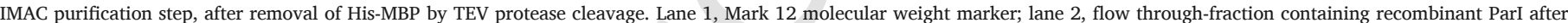
tag-removal. The theoretical molecular weight of ParI is calculated to be $54 \mathrm{kDa}$.

ing to cell death. This result supports the notion that recombinant ParI is active in vivo in E. coli. To ensure that the lack of cell growth was not a consequence of low competence or poor viability of the BL21Star (DE3) cell stock, transformations were attempted with plasmids encoding several unrelated proteins, and in all cases these produced colonies.

To investigate the McrBC sensitivity of DNA from the native host of ParI, P. arcticus 273-4 genomic DNA was isolated as well as genomic DNA from the related, but C5-DNA-MTase deficient $P$. sp. PRwf-1 strain. After treatment with McrBC restriction enzyme, genomic DNA from $P$. sp. PRwf-1 was found to be intact while genomic DNA from $P$. arcticus 273-4 appeared to be significantly degraded (Fig. 6). This McrBC sensitivity indicated that genomic DNA from $P$. arcticus 273-4 was methylated in vivo. According to REBASE, $P$. arcticus contains a second C5-DNA-MTase in addition to ParI, encoded by the gene psyc_0401 (M.ParAORF401P, Table 1). It is possible that this enzyme is responsible for the McrBC-sensitive methylation we observed. However, in light of previous results using recombinant His-ParI (Fig. 6) we can assume that native ParI is able to fully or partially methylate the $P$. arcticus 273-4 genomic DNA, thus, making it sensitive to McrBC. Thus, the results strongly indicate that ParI is a methyltransferase capable of methylating genomic DNA both in its native host and in a heterologous host.

\subsection{Recombinant ParI has methyltransferase activity in vitro}

In vitro DNA methyltransferase activity was examined by measuring the incorporation of the $3 \mathrm{H}$-methyl group from S-adenosylmethionine (3H-SAM) into DNA using a filter-binding assay at room temperature
(Fig. 7). Three versions of the purified protein were tested (MBP-ParI, His-ParI, and ParI after tag-removal) as well as cell lysates of MBP-ParI and His-ParI. For the samples from purified proteins, MBP-ParI is most active, but admittedly this affinity-purified sample has minor impurities and as such there is a small possibility of contaminant activity. Purified His-ParI also showed significant activity. No methyltransferase activity was observed with untagged ParI (the activity of untagged ParI was comparable to the blank in 4 out of 5 purification batches of protein tested with this method). The results indicate that loss of catalytic activity occurred during the purification steps necessary for tag removal. In Fig. 7, the results for the clarified lysates of MBP-ParI (MBP-ParI lysate) and His-ParI (His-ParI lysate) are included as orange bars, but as the specific ParI-concentrations in these samples are unknown, these results should not be over interpreted and strictly speaking can only be used as a qualitative verification of the presence of a DNA-MTase in the samples; however, they accord well with the results for purified proteins.

Prior to this, attempts were made to assess the in vitro activity and sequence specificity for untagged ParI methylation using a bisulfite assay; however, the results obtained by this method were inconclusive. Based on the results from the filter binding assay, this was most likely due to the absence of activity for this construct.

\section{Discussion}

ParI, the first C5-DNA-MTase from a psychrophilic bacterium to be characterized, displays DNA methylation activity in vivo and in vitro. Its biophysical characteristics are consistent with large regions of disorder 

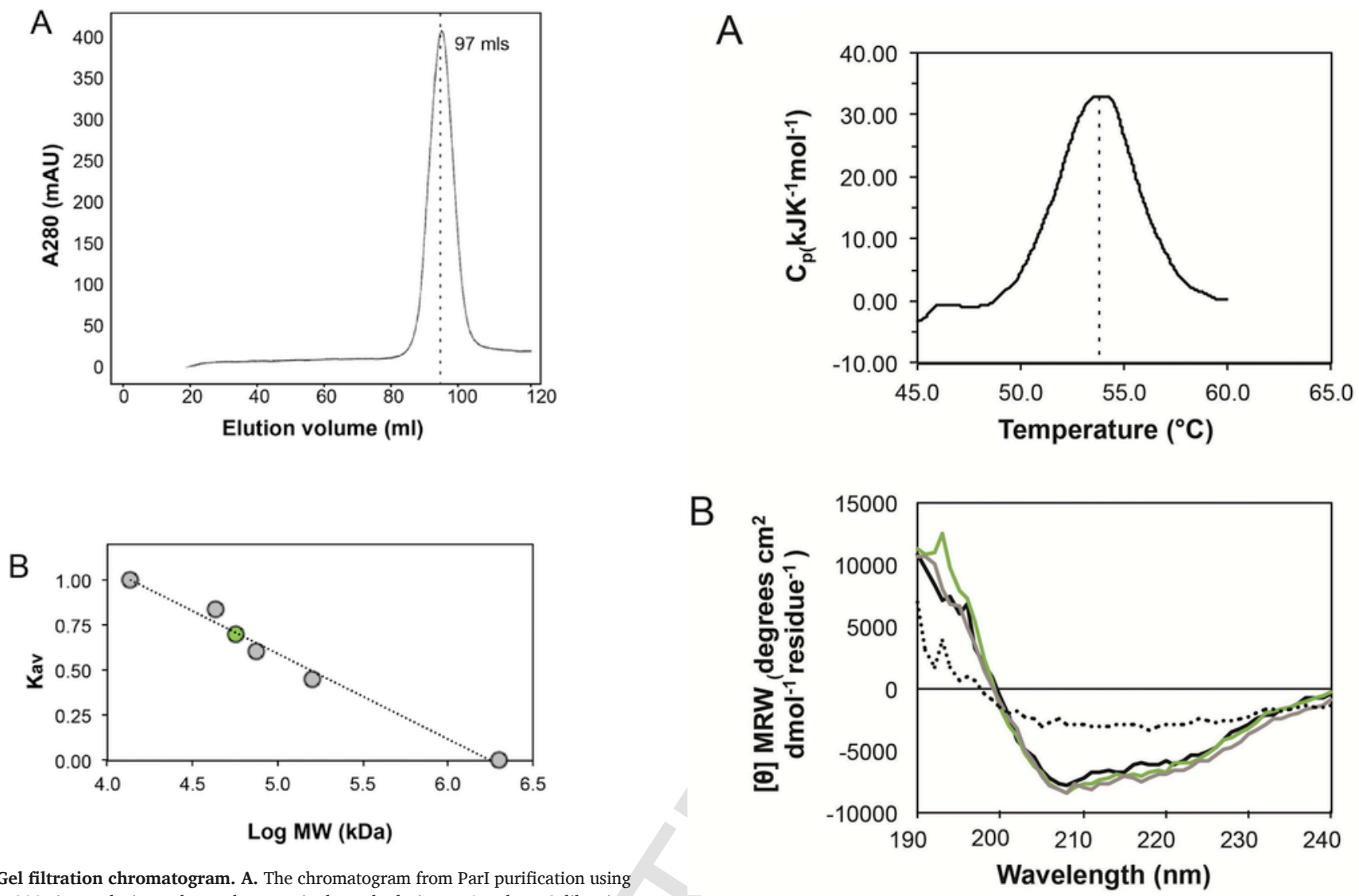

Fig. 4. Gel filtration chromatogram. A. The chromatogram from ParI purification using Superdex 200 size exclusion column shows a single-peak elution at $97 \mathrm{ml}$. B. Calibration curve of the column with $\log \mathrm{Mw}$ for ParI (green circle). The converted value is $57.5 \mathrm{kDa}$, which compares well with the calculated molecular weight for ParI of $54 \mathrm{kDa}$. (For interpretation of the references to colour in this figure legend, the reader is referred to the Web version of this article.)

including thermal unfolding transitions over broad temperature range and low percentage of secondary structure as measured by CD. ParI unfolds at temperatures typical of many mesophilic proteins, but its temperature optimum could not be determined due to apparent loss of activity after purification. The in vivo assays demonstrate toxicity of ParI when recombinantly expressed in $m c r+E$. coli at $37^{\circ} \mathrm{C}$, while assays in the native host were carried out at $25^{\circ} \mathrm{C}$, indicating that the enzyme is functional to some extent at both temperatures. Methyltransferase activity was also detected in vitro using radiolabelled 3H-SAM and pUC19 as a substrate. Activity was detected both in cell lysates and purified

Fig. 5. Thermal stability and unfolding of ParI. A. DSC thermogram for ParI after subtraction of the buffer baseline. The protein was scanned at a rate of $1^{\circ} \mathrm{C} / \mathrm{min}$ across a temperature range of $5-75^{\circ} \mathrm{C}$; here, only the relevant range for the unfolding event $\left(44-62^{\circ} \mathrm{C}\right)$ is shown. The graph was made in SigmaPlot. B. Secondary structure information rendered from $\mathrm{CD}$ spectra at $15^{\circ} \mathrm{C}$ in the presence of SAM (black line) and in the absence of SAM (green line), as well as at $35^{\circ} \mathrm{C}$ (gray line) and at $65^{\circ} \mathrm{C}$ (dotted line). (For interpretation of the references to colour in this figure legend, the reader is referred to the Web version of this article.)

samples, but not after tag removal. The higher activity of MBP-ParI compared to His-ParI, coupled with the complete loss of activity upon MBP removal suggests that the higher specific activity is likely due to increased ParI solubility for this construct. An intriguing point is that, although untagged ParI lost activity after MBP removal and subsequent purification steps, it appears to retain its overall structure. ParI has PCQ-to-DCK substitution in motif IV compared with homologous

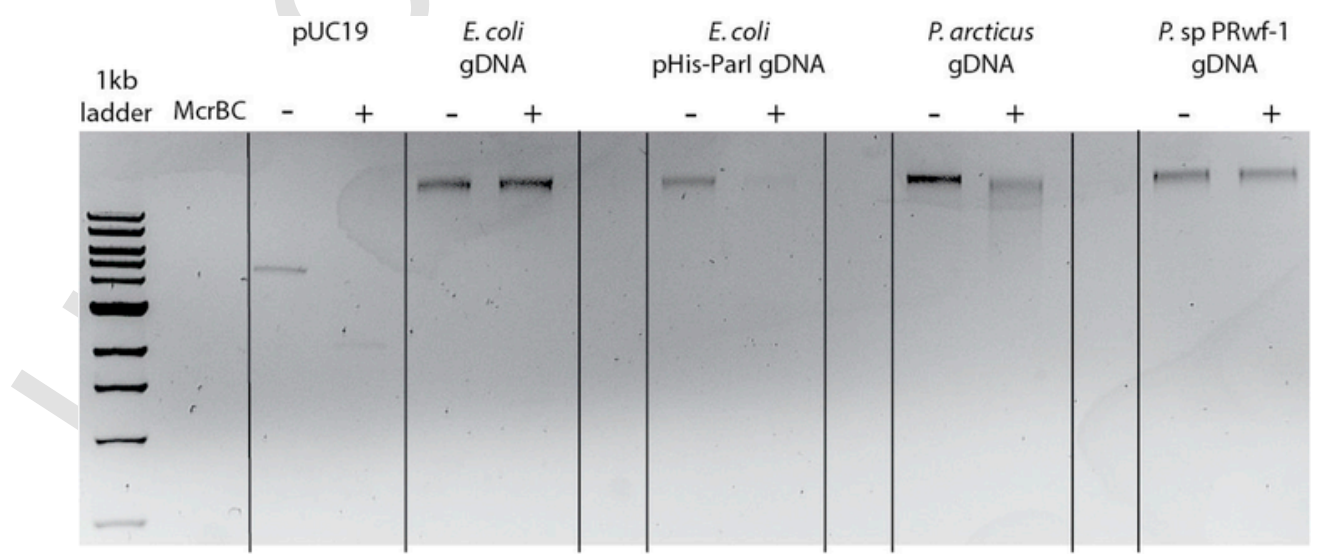

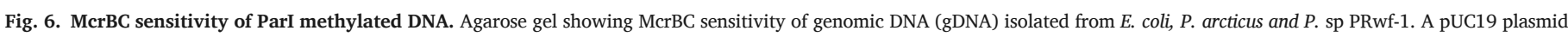

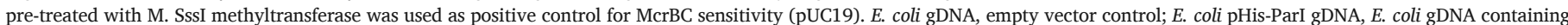
pHis-ParI; P. arcticus gDNA, gDNA from the parI-containing $P$. arcticus; P. sp PRwf-1, gDNA from the parI-deficient $P$. sp. Prwf-1. 


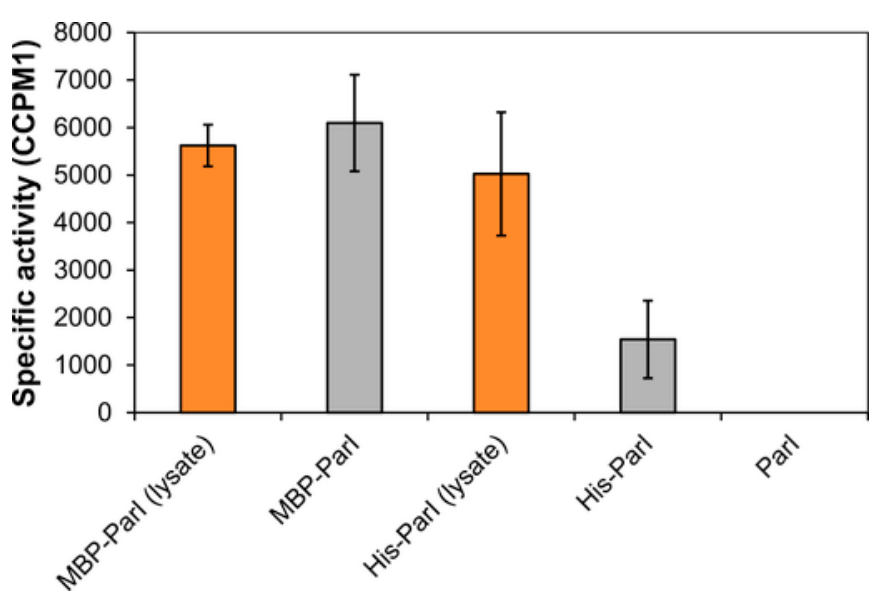

Fig. 7. Methyltransferase activity assay with radiolabelled SAM (3H-SAM). Bar graph showing incorporation of 3H-SAM into DNA by ParI constructs. The measured signal is expressed as blank- and concentration-corrected specific activity in CCPM1 (corrected counts per minute). MBP-ParI (lysate) and His-ParI (lysate) are clarified lysates of soluble protein, while MBP-ParI, His-ParI and ParI are purified proteins. The lysate samples are shown in orange. There is an uncertainty in the ParI-concentrations of the lysate samples, and as such these values can only be used qualitatively.

MTases, where the proline has a role in orientation of the activated cytosine and SAM in order to achieve methyl group transfer in the catalytic reaction [49]. Alterations in this region may make ParI particularly prone to inactivation, while remaining in a somewhat folded state.

Homology searches using BLAST revealed that ParI shares the highest sequence similarity (64\%) to an uncharacterized C5-DNA-MTase from $A$. baumannii, which is also of phage origin and shares the motif IV PCQ-to-DCK substitution.

The prevailing scenario for acquisition of orphan MTases is that during evolution, an MTase may have been part of a functional type II RM system that over time lost its REase member due to redundancy [50]. MTases are generally well-conserved during evolution as they methylate at the same or an overlapping site as members of other type II RM system found in bacteria, thereby complementing methylation and protection of host DNA by these enzymes [50]. A similar situation is found in the RM system of $E$. coli where Dcm, an orphan C5-DNA-MTase, has the same recognition sequence as the EcoRII RM system (CCWGG), and it is postulated that Dcm serves as back up for methylation of $E$. coli DNA $[50,51]$. Our hypothesis however, is that ParI was horizontally acquired as an orphan. Based on our bioinformatics investigations, as well as previously published data [18] we suggest that the gene encoding ParI is of phage origin, most likely a horizontally acquired gene from another bacterium during phage integration, with $A$. baumannii being a possible donor, although the original source still remains elusive. The parI gene is situated between two putative phage genes within the $P$. arcticus genome. The gene with the locus tag Psyc_0979, which is upstream to parI, is recognized by a BLAST homology search as part of temperate bacteriophage Psymv2 isolated from Psychrobacter sp. MV2, whose closest relative is an uncharacterized putative prophage within the $P$. arcticus 273-4 and A. baumannii genomes [43]. The region downstream to parI, encoding the gene with the locus tag Psyc_0981 is similar to the MU phage [18]. In addition to explaining the orphan status of ParI, horizontal gene transfer of parI into $P$. arcticus by a phage may explain why ParI does not exhibit typical cold-adapted features previously documented for other Psychrobacter enzymes such as the branched-chain 2-keto acid decarboxylase and murein peptide ligase which have optimal temperature of activity at $30^{\circ} \mathrm{C}[52,53]$.

We also note that $P$. arcticus $273-4$ is predicted to possesses an incomplete type IV McrBC-like REase, where the C subunit of the canoni- cal BC complex were missing, making the McrB non-functional $[54,55]$. An evolutionary explanation for this may be that the substrate for a functional McrBC enzyme is methylated cytosine and, assuming that the ParI enzyme is functional, an active McrBC would restrict the methylated genomic DNA, leading to cell death. Therefore the McrBC-negative background of $P$. arcticus made it possible to retain a horizontally transferred methyltransferase. This would be consistent with the results of our activity assays, which showed both that ParI-methylated DNA is sensitive to cleavage by McrBC enzymes, and that transformation with a plasmid encoding ParI was lethal to a McrBC-containing bacterial strain. In addition, the C5-DNA-MTase deficient $P$. sp. PRwf- 1 has a putative complete McrBC restriction system encoded in its genome.

\section{Conclusions}

In this paper we describe the recombinant expression and characterization of ParI, a C5-DNA-MTase from P. arcticus 273-4. To our knowledge, this is the first characterization of an orphan C5-DNA-MTase from a psychrophilic bacterium. The C5-DNA-MTase could not be expressed in regular E. coli expression strains, likely due to the presence of McrBC enzymes that restricted methylated DNA. Successful expression was obtained using an McrBC-negative E. coli expression strain. To improve solubility, an MBP tag was added as a fusion partner between the His-tag and ParI. This contributed to an overall increased yield and solubility. Despite originating from $P$. arcticus 273-4, ParI did not exhibit expected cold-adapted features such as a low melting temperature. We hypothesize that the reason for this is ParI's origin from a phage that is not cold-adapted.

Using two different in vivo assays where ParI-methylated genomic DNA was treated with McrBC, we showed that both native and recombinant ParI is able to methylate genomic DNA. Using a radiolabeled methyltransferase assay, we showed that ParI possesses methyltransferase activity also in vitro but only as a fusion construct with MBP and/ or His-tag, while the untagged protein showed no activity.

\section{Declarations}

Ethics approval and consent to participate

Not applicable.

Consent to publish

Not applicable.

\section{Availability of data and materials}

All data generated or analysed during this study are included in this published article and its supplementary information files.

\section{Competing interests}

None to be declared.

Funding

This study was supported by UiT The Arctic University of Norway.

\section{Authors' contributions}

IL, BA and GEKB conceived the study and participated in its design and coordination, helped in data analysis and reviewed the manuscript, MG performed all experiments, interpreted the data and drafted the 
manuscript; AW helped in experimental design, data analysis, performed the $\mathrm{CD}$ experiment and reviewed the manuscript. All authors read and approved the final manuscript.

\section{Acknowledgements}

This work has been funded by an internal $\mathrm{PhD}$ grant at UiT The Arctic University of Norway.

\section{Appendix A. Supplementary data}

Supplementary data related to this article can be found at https:// doi.org/10.1016/j.pep.2018.05.012.

\section{References}

[1] A. Jeltsch, Beyond Watson and Crick: DNA methylation and molecular enzymology of DNA methyltransferases, Chembiochem 3 (2002) 274-293.

[2] A. Jeltsch, R.Z. Jurkowska, T.P. Jurkowski, K. Liebert, P. Rathert, M. Schlickenrieder, Application of DNA methyltransferases in targeted DNA methylation, Appl. Microbiol. Biotechnol. 75 (2007) 1233-1240.

[3] X. Cheng, S. Kumar, J. Posfai, J.W. Pflugrath, R.J. Roberts, Crystal structure of the Hhal DNA methyltransferase complexed with S-adenosyl-L-methionine, Cell 74 (1993) 299-307.

[4] G.G. Wilson, Organization of restriction-modification systems, Nucleic Acids Res. 19 (1991) 2539-2566.

[5] R.J. Roberts, T. Vincze, J. Posfai, D. Macelis, REBASE -a database for DNA restriction and modification: enzymes, genes and genomes, Nucleic Acids Res. 38 (2009) D234-D236.

[6] G.G. Wilson, Type II restriction-modification systems, Trends Genet. 4 (1988) 314-318.

[7] J. Murphy, J. Mahony, S. Ainsworth, A. Nauta, D. van Sinderen, Bacteriophage orphan DNA methyltransferases: insights from their bacterial origin, function, and occurrence, Appl. Environ. Microbiol. 79 (2013) 7547-7555.

[8] A. Reisenauer, L.S. Kahng, S. McCollum, L. Shapiro, Bacterial DNA methylation: a cell cycle regulator?, J. Bacteriol. 181 (1999) 5135-5139.

[9] E.S. Gromova, A.V. Khoroshaev, Prokaryotic DNA methyltransferases: the structure and the mechanism of interaction with DNA, Mol. Biol. 37 (2003) 260-272.

[10] A. Løbner-Olesen, O. Skovgaard, M.G. Marinus, Dam methylation: coordinating cellular processes, Curr. Opin. Microbiol. 8 (2005) 154-160.

[11] D.M. Heithoff, R.L. Sinsheimer, D.A. Low, M.J. Mahan, An essential role for DNA adenine methylation in bacterial virulence, Science 284 (1999) 967-970.

[12] K. Vasu, V. Nagaraja, Diverse functions of restriction-modification systems in addition to cellular defense, Microbiol. Mol. Biol. Rev. 77 (2013) 53-72.

[13] J. Song, O. Rechkoblit, T.H. Bestor, D.J. Patel, Structure of DNMT1-DNA complex reveals a role for autoinhibition in maintenance DNA methylation, Science 331 (2011) 1036-1040.

[14] S. Kumar, X. Cheng, S. Klimasauskas, S. Mi, J. Posfai, R.J. Roberts, G.G. Wilson, The DNA (cytosine-5) methyltransferases, Nucleic Acids Res. 22 (1994) 1.

[15] K.M. Reinisch, L. Chen, G.L. Verdine, W.N. Lipscomb, The crystal structure of Haelll methyltransferase covalently complexed to DNA: an extrahelical cytosine and rearranged base pairing, Cell 82 (1995) 143-153.

[16] U.T. Sankpal, D.N. Rao, Structure, function, and mechanism of HhaI DNA methyltransferases, Crit. Rev. Biochem. Mol. Biol. 37 (2002) 167-197.

[17] T.A. Vishnivetskaya, M.A. Petrova, J. Urbance, M. Ponder, C.L. Moyer, D.A Gilichinsky, J.M. Tiedje, Bacterial community in ancient Siberian permafrost as characterized by culture and culture-independent methods, Astrobiology 6 (2006) $400-414$.

[18] H.L. Ayala-del-Río, P.S. Chain, J.J. Grzymski, M.A. Ponder, N. Ivanova, P.W. Bergholz, G. Di Bartolo, L. Hauser, M. Land, C. Bakermans, The genome sequence of Psychrobacter arcticus 273-4, a psychroactive Siberian permafrost bacterium, reveals mechanisms for adaptation to low-temperature growth, Appl. Environ. Microbiol. 76 (2010) 2304-2312.

[19] C. Bakermans, H.L. Ayala-del-Rio, M.A. Ponder, T. Vishnivetskaya, D. Gilichinsky, M.F. Thomashow, J.M. Tiedje, Psychrobacter cryohalolentis sp. nov. and Psychrobacter arcticus sp. nov., isolated from Siberian permafrost, Int. J. Syst. Evol. Microbiol. 56 (2006) 1285-1291.

[20] S. Zhang, W. Song, M. Yu, X. Lin, Comparative genomics analysis of five Psychrobacter strains isolated from world-wide habitats reveal high intra-genus variations, Extremophiles 21 (2017) 581-589.

[21] P.W. Bergholz, C. Bakermans, J.M. Tiedje, Psychrobacter arcticus 273-4 uses resource efficiency and molecular motion adaptations for subzero temperature growth, J. Bacteriol. 191 (2009) 2340-2352.

[22] M. Tomkuviené, E. Kriukienè, S. Klimašauskas, DNA labeling using DNA methyltransferases, in: A. Jeltsch, R.Z. Jurkowska (Eds.), DNA Methyltransferases - Role and Function, Springer International Publishing, 2016, pp. 511-535, Cham.

[23] S. Klimašauskas, E. Weinhold, A new tool for biotechnology: AdoMet-dependent methyltransferases, Trends Biotechnol. 25 (2007) 99-104.
[24] J. Deen, C. Vranken, V. Leen, R.K. Neely, K.P.F. Janssen, J. Hofkens, Methyltrans ferase-directed labeling of biomolecules and its applications, Angew. Chem. Int. Ed. 56 (2017) 5182-5200.

[25] C. Notredame, D.G. Higgins, J. Heringa, T-Coffee: a novel method for fast and accurate multiple sequence alignment, J. Mol. Biol. 302 (2000) 205-217.

[26] X. Robert, P. Gouet, Deciphering key features in protein structures with the new ENDscript server, Nucleic Acids Res. 42 (2014) W320-W324.

[27] D.T. Jones, Protein secondary structure prediction based on position-specific scoring matrices, JMB (J. Mol. Biol.) 292 (1999) 195-202.

[28] Y. Zhou, Y. Liang, K.H. Lynch, J.J. Dennis, D.S. Wishart, PHAST: a fast phage search tool, Nucleic Acids Res. (2011).

[29] V. Solovyev, A. Salamov, Automatic annotation of microbial genomes and metagenomic sequences, Metagenomics and its applications in agriculture, biomedicine and environmental studies (2011) 61-78.

[30] T.J. Macke, D.J. Ecker, R.R. Gutell, D. Gautheret, D.A. Case, R. Sampath, RNAMotif, an RNA secondary structure definition and search algorithm, Nucleic Acids Res. 29 (2001) 4724-4735.

[31] D. Gautheret, A. Lambert, Direct RNA motif definition and identification from multiple sequence alignments using secondary structure profiles, J. Mol. Biol. 313 (2001) 1003-1011.

[32] F. Katzen, Gateway ${ }^{\circledR}$ Recombinational Cloning: a Biological Operating System, 2007.

[33] S. Boivin, S. Kozak, R. Meijers, Optimization of protein purification and characterization using Thermofluor screens, Protein Expression and Purification 91 (2013) 192-206.

[34] S.W. Provencher, J. Gloeckner, Estimation of globular protein secondary structure from circular dichroism, Biochemistry 20 (1981) 33-37.

[35] N. Sreerama, R.W. Woody, A self-consistent method for the analysis of protein secondary structure from circular dichroism, Anal. Biochem. 209 (1993) 32-44.

[36] I.H.M. Van Stokkum, H.J.W. Spoelder, M. Bloemendal, R. Van Grondelle, F.C.A. Groen, Estimation of protein secondary structure and error analysis from circular dichroism spectra, Anal. Biochem. 191 (1990) 110-118.

[37] N. Sreerama, S.Y.U. Venyaminov, R.W. Woody, Estimation of the number of helical and strand segments in proteins using circular dichroism spectroscopy, Protein Sci. 8 (1999) 370-380.

[38] A. Laisne, D. Pompon, J.L. Leroy, [C7GC4]4 association into supra molecular i-motif structures, Nucleic Acids Res. 38 (2010) 3817-3826.

[39] L. Whitmore, B.A. Wallace, Protein secondary structure analyses from circular dichroism spectroscopy: methods and reference databases, Biopolymers 89 (2008) 392-400.

[40] N. Sreerama, S.Y. Venyaminov, R.W. Woody, Estimation of protein secondary structure from circular dichroism spectra: inclusion of denatured proteins with native proteins in the analysis, Anal. Biochem. 287 (2000) 243-251.

[41] N. Sreerama, R.W. Woody, A self-consistent method for the analysis of protein secondary structure from circular dichroism, Anal. Biochem. 209 (1993) 32-44.

[42] U.T. Sankpal, D.N. Rao, Mutational analysis of conserved residues in HhaI DNA methyltransferase, Nucleic Acids Res. 30 (2002) 2628-2638.

[43] T.L. Meiring, I.M. Tuffin, C. Cary, D.A. Cowan, Genome sequence of temperate bacteriophage Psymv2 from Antarctic Dry Valley soil isolate Psychrobacter sp. MV2, Extremophiles 16 (2012) 715-726.

[44] R. Rossau, A. Van landschoot, M. Gillis, J. De ley, Taxonomy of Moraxellaceae fam. Nov., a new bacterial family to accommodate the genera Moraxella, acinetobacter, and psychrobacter and related organisms, Int. J. Syst. Bacteriol. 41 (1991) 310-319.

[45] E.A. Raleigh, N.E. Murray, H. Revel, R.M. Blumenthal, D. Westaway, A.D. Reith, P.W.J. Rigby, J. Elhai, D. Hanahan, McrA and McrB restriction phenotypes of some E. coli strains and implications for gene cloning, Nucleic Acids Res. 16 (1988) 1563-1575.

[46] P. Sun, J.E. Tropea, D.S. Waugh, Enhancing the Solubility of Recombinant Protein in Escherichia coli by Using Hexahistidine-tagged Maltose-binding Protein as a Fusion Partner, Heterologous Gene Expression in E Coli, Springer, 2011259-274.

[47] A. Williamson, H. Pedersen, Recombinant expression and purification of an ATP-dependent DNA ligase from Aliivibrio salmonicida, Protein Expression and Purification 97 (2014) 29-36.

[48] N.J. Greenfield, Using circular dichroism spectra to estimate protein secondary structure, Nat. Protoc. 1 (2006) 2876-2890.

[49] V.G. Kossykh, S.L. Schlagman, S. Hattman, Function of Pro-185 in the ProCys of conserved motif IV in the EcoRII [cytosine-C5]-DNA methyltransferase, FEBS Letters 370 (1995) 75-77.

[50] A.S.N. Seshasayee, P. Singh, S. Krishna, Context-dependent conservation of DNA methyltransferases in bacteria, Nucleic Acids Res. 40 (2012) 7066-7073.

[51] K.T. Militello, R.D. Simon, M. Qureshi, R. Maines, M.L. Van Horne, S.M. Hennick S.K. Jayakar, S. Pounder, Conservation of Dcm-mediated cytosine DNA methylation in Escherichia coli, FEMS Microbiology Letters 328 (2012) 78-85.

[52] D. Das, M. Hervé, J. Feuerhelm, C.L. Farr, H.-J. Chiu, M.-A. Elsliger, M.W. Knuth H.E. Klock, M.D. Miller, A. Godzik, Structure and function of the first full-length murein peptide ligase $(\mathrm{Mpl})$ cell wall recycling protein, PLoS One 6 (2011), e17624.

[53] J. Wei, J.G. Timler, C.M. Knutson, B.M. Barney, Branched-chain 2-keto acid decarboxylases derived from Psychrobacter, FEMS Microbiology Letters 346 (2013) 105-112.

[54] E.A. Raleigh, Organization and function of the mcrBC genes of Escherichia coli K12, Mol. Microbiol. 6 (1992) 1079-1086.

[55] E. Sutherland, L. Coe, E.A. Raleigh, McrBC: a multisubunit GTP-dependent restriction endonuclease, J. Mol. Biol. 225 (1992) 327-348. 